\title{
A DEEP MULTICOLOR SURVEY. VI. NEAR-INFRARED OBSERVATIONS, SELECTION EFFECTS, AND NUMBER COUNTS ${ }^{1}$
}

\author{
PaUl MarTini \\ Department of Astronomy, Ohio State University, 140 West Eighteenth Avenue, Columbus, OH 43210; martini@astronomy.ohio-state.edu \\ Received 2000 August 22 ; accepted 2000 October 17
}

\begin{abstract}
I present near-infrared $J(1.25 \mu \mathrm{m}), H(1.65 \mu \mathrm{m})$, and $K(2.2 \mu \mathrm{m})$ imaging observations of $185 \operatorname{arcmin}^{2}$ in 21 high galactic latitude fields. These observations reach limiting magnitudes of $J \sim 21, H \sim 20$, and $K \sim 18.5 \mathrm{mag}$. The detection efficiency, photometric accuracy, and selection biases as a function of integrated object brightness, size, and profile shape are quantified in detail. I evaluate several popular methods for measuring the integrated light of faint galaxies and show that only aperture magnitudes provide an unbiased measure of the integrated light that is independent of apparent magnitude. These $J$, $H$, and $K$ counts and near-infrared colors are in best agreement with passive galaxy formation models with at most a small amount of merging (for $\Omega_{M}=0.3$ and $\Omega_{\Lambda}=0.7$ ).

Key words: cosmology: observations - galaxies: evolution - galaxies: formation galaxies: photometry
\end{abstract}

\section{INTRODUCTION}

The surface density of galaxies as a function of integrated brightness, also known as the number-magnitude relation, is one of the classic tests of observational cosmology. This test has a long, distinguished history as a tool for studying the nature of the universe and the evolution of galaxies (e.g., Hubble 1934; Sandage 1961; Tinsley 1977). Surveys to progressively deeper apparent magnitude limits have measured the number-magnitude relation at a range of wavelengths, and this work has been reviewed by Koo \& Kron (1992) and Ellis (1997). The observed number-magnitude relations are compared with the predictions of galaxy evolution models, such as those by Yoshii \& Takahara (1988), Guiderdoni \& Rocca-Volmerange (1990), and Gardner (1998), to determine cosmological parameters and study galaxy formation and evolution.

Near-infrared (NIR; $1-2.5 \mu \mathrm{m}$ ) galaxy counts are a valuable addition to studies of galaxy formation and evolution with the number-magnitude relation. The spectral energy distributions (SEDs) of most galaxies are relatively constant as a function of wavelength in the NIR because this wavelength regime is dominated by light from old, evolved stars. The shape and uniformity of galaxy SEDs in the NIR results in relatively small $k$-corrections for galaxies up to $z \sim 1$, and therefore the NIR luminosity is representative of the total stellar luminosity. A recent burst of star formation, in contrast, can significantly increase the brightness of a galaxy at visible and UV wavelengths. This may cause a galaxy with a large amount of current star formation to appear as bright as a more quiescent galaxy with a much larger stellar mass. The presence of a great deal of dust will also attenuate the brightness of galaxies at these wavelengths and further complicate the relation between UV or visible luminosity and total stellar luminosity. NIR number counts to a limit of $\sim 20 \mathrm{mag}$, which are dominated by galaxies at $z<1$, provide a less uncertain means of evaluating models of galaxy formation. The old, evolved stars that dominate the NIR light are also good tracers of the

\footnotetext{
${ }^{1}$ Based on observations obtained at MDM Observatory, operated by Columbia University, Dartmouth College, the University of Michigan, and the Ohio State University.
}

total stellar mass, and therefore NIR measurements are an excellent method for direct study of the stellar mass evolution in galaxies in the context of hierarchical galaxy formation (Brinchmann \& Ellis 2000).

NIR number counts to date have mostly been obtained at K (Gardner, Cowie, \& Wainscoat 1993; Cowie et al. 1994; Djorgovski et al. 1995; McLeod et al. 1995; Gardner et al. 1996; Moustakas et al. 1997) as this is the longestwavelength atmospheric transmission window with sufficient sensitivity to detect high-redshift galaxies in a reasonable time. Faint counts have also been obtained at $H$ (Teplitz et al. 1998; Yan et al. 1998) with the Near Infrared Camera and Multi-Object Spectrometer (NICMOS) on the Hubble Space Telescope (HST), though given its small field of view there are few bright galaxies in this sample. Some observations have also been obtained at both $J$ and $K$ (Bershady, Lowenthal, \& Koo 1998; Teplitz, McLean, \& Malkan 1999; Väisänen et al. 2000), and these studies have used the mean NIR colors as a function of apparent magnitude as an additional constraint for galaxy evolution models.

I have obtained $J, H$, and $K$ observations of $185 \operatorname{arcmin}^{2}$ of high galactic latitude fields that extend to $J \sim 21$, $H \sim 20$, and $K \sim 18.5$. The median redshift to $K=18.5$ is $z \sim 0.5$ in the $K$-selected Hawaii Redshift Survey (Cowie et al. 1996). These observations are made up of 21 subfields of the Deep Multicolor Survey (DMS) fields observed by Hall et al. (1996a). The DMS covers a total area of $0.83 \mathrm{deg}^{2}$ in six filters: $U, B, V, R, I_{75}$, and $I_{86}$. The DMS is composed of six different fields, and to date this rich data set has been used to study the quasar luminosity function (Hall et al. 1996b; Kennefick et al. 1997), the evolution of blue galaxies (Liu et al. 1998), and the M dwarf distribution, mass, and luminosity function (Martini \& Osmer 1998). The selection criteria for the DMS fields were relatively low galactic foreground extinction and avoidance of very bright stars. Five of the fields are equatorial to facilitate follow-up spectroscopy from both hemispheres.

In $\S 2$, I describe the NIR observations, while in $\S 3$, I discuss the image processing, photometric solution, and catalogs. I discuss the detection efficiencies in $\S 4$, galaxy photometry in $\S 5$, and star-galaxy separation in $\S 6$. The 
galaxy number counts and NIR colors of these galaxies are presented in $\$ \S 7$ and 8 . In a separate paper (Martini 2001) I combine these NIR observations with the $U, B, V, R, I_{75}$, and $I_{86} \mathrm{CCD}$ data to compute photometric redshifts for this NIR sample to study the visible-NIR colors of these galaxies, particularly the extremely red objects.

\section{OBSERVATIONS}

A total of 21 randomly selected subfields of the DMS were observed through the NIR $J, H$, and $K$ filters. These images were obtained with TIFKAM ${ }^{2}$ (Pogge et al. 1998), an NIR imager/spectrograph with a $512 \times 1024 \mathrm{InSb}$ detector on the $2.4 \mathrm{~m}$ Hiltner telescope of the MDM Observatory. The $\mathrm{f} / 7$ camera was used for all the observations; this camera has a plate scale of 0.3 pixel $^{-1}$ on the Hiltner telescope. Table 1 is a log of the observations, listing the date each field was observed in each filter and the seeing measured in that field-filter combination. Each of the 21 fields was observed for a total of 60 minutes of on-source integration time per filter. The observations were obtained in a six-point dither pattern with a $10^{\prime \prime}-15^{\prime \prime}$ offset between positions. Individual exposures in the dither pattern were 1 to 2 minutes and comprised a combination of integration time and co-adds such that the sky level in the frames was less than 10,000 counts. Tests of the detector linearity showed that the array is less than $1 \%$ nonlinear at this count level.

\footnotetext{
${ }^{2}$ TIFKAM: The Instrument Formerly Known As MOSAIC. This instrument is also known as ONIS at Kitt Peak.
}

Dark frames were taken nearly every night with the same combination of exposure time and number of co-adds as that of the science frames.

Standard stars from Persson et al. (1998) were observed each night at a range of air masses. A series of red stars was also observed to determine the transformation of the instrumental magnitude system to the Cerro Tololo InterAmerican Observatory (CTIO) CIT photometric system (Elias et al. 1982). Red stars were observed at least one night per observing run as there were several changes to the filters over the course of the observations for this program. These changes are discussed below in $\S 3.2$. The standard stars were observed in a five- or six-point dither pattern with $15^{\prime \prime}$ offsets, though with only $15-30 \mathrm{~s}$ of on-source integration per dither position.

\section{IMAGE PROCESSING}

\subsection{Survey Fields}

All the data were reduced with IRAF $^{3}$ and many of the data processing steps took advantage of the PHIIRS ${ }^{4}$ package, a collection of IRAF routines compiled by Pat Hall. The first step in the data processing was subtraction of a dark frame from each image. The individual images were

${ }^{3}$ IRAF is distributed by the National Optical Astronomy Observatories, which are operated by the Association of Universities for Research in Astronomy, Inc., under cooperative agreement with the National Science Foundation.

${ }^{4}$ Available at http://iraf.noao.edu/iraf/web/contrib.html.

TABLE 1

LOG OF OBSERVATIONS OF THE 21 SUBFIELDS OF THE DMS

\begin{tabular}{|c|c|c|c|c|c|c|c|}
\hline \multirow[b]{2}{*}{$\begin{array}{l}\text { FIELD } \\
\text { (1) }\end{array}$} & \multirow[b]{2}{*}{$\begin{array}{c}\text { AREA } \\
\text { (2) }\end{array}$} & \multicolumn{3}{|c|}{ DATE } & \multicolumn{3}{|c|}{ FWHM } \\
\hline & & $\begin{array}{c}J \\
(3)\end{array}$ & $\begin{array}{l}H \\
(4)\end{array}$ & $\begin{array}{l}K \\
(5)\end{array}$ & $\begin{array}{c}J \\
(6)\end{array}$ & $\begin{array}{l}H \\
(7)\end{array}$ & $\begin{array}{l}K \\
(8)\end{array}$ \\
\hline CF1.. & $4.52^{\mathrm{a}}$ & 1999 Sep 28 & 1999 Sep 29 & 1999 Sep 29 & 1.4 & 1.6 & 1.5 \\
\hline CF3....... & 6.94 & 1997 Oct 14 & 1997 Oct 16 & 1997 Oct 14 & 1.8 & 1.2 & 1.8 \\
\hline $21 \mathrm{WC} \ldots \ldots \ldots \ldots$ & 7.74 & 1997 Oct 15 & 1999 Sep 26 & 1999 Sep 26 & 1.4 & 1.4 & 1.3 \\
\hline $22 \mathrm{EC} \ldots \ldots \ldots \ldots$ & 7.46 & 1997 Oct 16 & 1997 Oct 17 & 1997 Oct 16 & 1.2 & 1.3 & 1.2 \\
\hline $10 \mathrm{EC} \ldots \ldots \ldots \ldots$ & 9.49 & 1998 Apr 11 & 1998 Apr 11 & 1998 Apr 10 & 1.5 & 1.4 & 1.2 \\
\hline $14 N C \ldots \ldots \ldots \ldots$ & 7.55 & 1999 May 15 & 1998 Apr 11 & 1998 Apr 10 & 1.2 & 1.5 & 1.2 \\
\hline $17 N C \ldots \ldots \ldots \ldots$ & 8.71 & 1998 Apr 11 & $1998 \mathrm{Apr} 15^{\mathrm{b}}$ & 1998 Apr 10 & 1.5 & 1.1 & 1.2 \\
\hline $14 \mathrm{SC} .$. & 9.17 & 1998 Apr $14^{\mathrm{c}}$ & $1998 \mathrm{Apr} 14^{\mathrm{c}}$ & $1998 \mathrm{Apr} 14^{\mathrm{c}}$ & 1.3 & 1.2 & 1.6 \\
\hline $22 \mathrm{WC} \ldots$. & 9.23 & 1998 Oct 03 & 1998 Oct 03 & 1998 Oct 03 & 1.6 & 1.6 & 1.2 \\
\hline 01EC60S .. & 8.81 & 1998 Oct 03 & 1998 Oct 02 & 1998 Oct 02 & 1.3 & 1.2 & 1.3 \\
\hline $01 \mathrm{WC} \ldots \ldots \ldots \ldots$ & 9.94 & 1998 Oct 03 & 1998 Oct 02 & 1998 Oct 02 & 1.3 & 1.3 & 1.5 \\
\hline $21 \mathrm{EC} \ldots \ldots \ldots \ldots$ & 9.31 & 1998 Oct 01 & 1998 Oct 01 & 1998 Oct 02 & 1.4 & 1.2 & 1.5 \\
\hline 01WC150W ....... & 9.86 & 1998 Oct 03 & 1998 Oct 03 & 1998 Oct 03 & 1.4 & 1.5 & 1.5 \\
\hline 21WC150W ...... & 9.30 & 1998 Oct 04 & 1998 Oct 04 & 1998 Oct 04 & 1.7 & 1.5 & 1.4 \\
\hline 14NC150W ...... & 9.04 & 1999 May 16 & 1999 May 16 & 1999 May 16 & 1.0 & 1.0 & 1.0 \\
\hline $14 \mathrm{NC} 150 \mathrm{E} \ldots \ldots$. & 9.36 & 1999 May 17 & 1999 May 17 & 1999 May 17 & 1.6 & 1.0 & 1.2 \\
\hline $14 \mathrm{NC} 300 \mathrm{~W} . . .$. & 9.90 & 1999 Мay 19 & 1999 Мау 19 & 1999 Мау 19 & 1.2 & 1.1 & 1.2 \\
\hline $14 \mathrm{NC} 300 \mathrm{E} . . . .$. & 9.54 & 1999 May 20 & 1999 Мay 20 & 1999 May 20 & 0.9 & 0.9 & 1.0 \\
\hline $17 \mathrm{SC} \ldots \ldots \ldots \ldots$ & 9.70 & 1999 May 15 & 1999 Мay 26 & 1999 May 27 & 1.1 & 1.1 & 1.1 \\
\hline 21WC150E ..... & 9.80 & 1999 Sep 28 & 1999 Sep 28 & 1999 Sep 28 & 1.3 & 1.3 & 1.3 \\
\hline 22EC150W ..... & 10.19 & 1999 Sep 29 & 1999 Sep 29 & 1999 Sep 29 & 1.7 & 1.8 & 1.9 \\
\hline
\end{tabular}

NoTE..- Col. (1) lists the field identifier, which indicates either the center of one of the fields listed in Hall et al. (1996a) or the offset from the center of one of these fields. For example, 21WC refers to the center of Field $21 \mathrm{~W}$ and $01 \mathrm{EC} 60 \mathrm{~S}$ refers to a field $60^{\prime \prime}$ south of Field 01E. The two exceptions to this naming convention are CF1 and CF3, which are defined in Hall et al. (1998). Col. (2) lists the area in square arcminutes of each subfield. Cols. (3)-(5) list the UT date of the observations in $J, H$, and $K$, respectively. Cols. (6)-(8) list the FWHM in arcseconds of the PSF in the final, combined frame for $J, H$, and $K$.

${ }^{a}$ Area that does not overlap 01WC150W.

${ }^{b}$ Calibration obtained 1998 Oct 3.

${ }^{c}$ Calibration obtained 1999 May 15. 
TABLE 2

PHOTOMETRIC SOLUTIONS

\begin{tabular}{|c|c|c|c|c|c|c|c|c|c|c|c|c|}
\hline $\begin{array}{l}\text { Date } \\
\text { (1) }\end{array}$ & $\begin{array}{l}j_{0} \\
(2)\end{array}$ & $\begin{array}{l}j_{1} \\
(3)\end{array}$ & $\begin{array}{l}j_{2} \\
\text { (4) }\end{array}$ & $\begin{array}{l}\sigma(\mathbf{J}) \\
(5)\end{array}$ & $\begin{array}{l}h_{0} \\
(6)\end{array}$ & $\begin{array}{l}h_{1} \\
(7)\end{array}$ & $\begin{array}{l}h_{2} \\
(8)\end{array}$ & $\begin{array}{l}\sigma(\mathrm{H}) \\
(9)\end{array}$ & $\begin{array}{c}k_{0} \\
(10)\end{array}$ & $\begin{array}{c}k_{1} \\
(11)\end{array}$ & $\begin{array}{c}k_{2} \\
(12)\end{array}$ & $\begin{array}{l}\sigma(\mathrm{K}) \\
(13)\end{array}$ \\
\hline 1997 Oct $11 \ldots \ldots$. & 22.204 & -0.040 & 0.160 & 0.046 & 21.961 & 0.000 & 0.060 & 0.074 & 21.245 & -0.060 & 0.000 & 0.127 \\
\hline 1997 Oct $14 \ldots \ldots$ & 22.303 & -0.047 & 0.160 & 0.036 & 22.040 & -0.006 & 0.060 & 0.048 & 21.290 & -0.060 & 0.000 & 0.048 \\
\hline 1997 Oct $15 \ldots \ldots$ & 22.351 & -0.038 & 0.160 & 0.048 & 22.076 & -0.021 & 0.060 & 0.029 & 21.354 & -0.053 & 0.000 & 0.077 \\
\hline 1997 Oct $16 \ldots \ldots$ & 22.346 & -0.040 & 0.160 & 0.036 & 22.063 & -0.040 & 0.060 & 0.034 & 21.324 & -0.060 & 0.000 & 0.064 \\
\hline 1997 Oct $17 \ldots \ldots$ & 22.330 & -0.040 & 0.160 & 0.031 & 22.070 & 0.000 & 0.060 & 0.035 & 21.320 & -0.060 & 0.000 & 0.039 \\
\hline 1998 Apr $10 \ldots \ldots$ & 22.425 & -0.113 & 0.060 & 0.034 & 22.033 & -0.058 & 0.060 & 0.036 & 21.316 & -0.050 & 0.000 & 0.035 \\
\hline 1998 Apr $11 \ldots \ldots$ & 22.409 & -0.206 & 0.060 & 0.022 & 22.047 & -0.158 & 0.060 & 0.028 & 21.311 & -0.134 & 0.000 & 0.048 \\
\hline 1998 Oct $01 \ldots \ldots$ & 22.293 & -0.060 & 0.060 & 0.032 & 22.075 & -0.037 & 0.000 & 0.021 & 21.372 & -0.065 & 0.000 & 0.032 \\
\hline 1998 Oct $02 \ldots \ldots$. & 22.336 & -0.070 & 0.060 & 0.019 & 22.147 & 0.000 & 0.000 & 0.035 & 21.485 & -0.052 & 0.000 & 0.017 \\
\hline 1998 Oct $03 \ldots \ldots$ & 22.355 & -0.081 & 0.060 & 0.032 & 22.164 & -0.026 & 0.000 & 0.020 & 21.485 & -0.091 & 0.000 & 0.033 \\
\hline 1998 Oct $04 \ldots \ldots$. & 22.332 & -0.102 & 0.060 & 0.021 & 22.165 & -0.058 & 0.000 & 0.022 & 21.470 & -0.113 & 0.000 & 0.024 \\
\hline 1999 May $15 \ldots . .$. & 22.377 & -0.090 & 0.060 & 0.040 & 22.182 & 0.000 & 0.000 & 0.017 & 21.494 & -0.050 & 0.000 & 0.029 \\
\hline 1999 May $16 \ldots . .$. & 22.412 & -0.090 & 0.060 & 0.032 & 22.181 & -0.008 & 0.000 & 0.025 & 21.505 & -0.046 & 0.000 & 0.019 \\
\hline 1999 Мay $17 \ldots \ldots$ & 22.389 & -0.090 & 0.060 & 0.023 & 22.175 & -0.032 & 0.000 & 0.034 & 21.495 & -0.043 & 0.000 & 0.026 \\
\hline 1999 May $19 . . . .$. & 22.430 & -0.090 & 0.060 & 0.023 & 22.173 & -0.031 & 0.000 & 0.029 & 21.494 & -0.083 & 0.000 & 0.036 \\
\hline 1999 May $20 \ldots . .$. & 22.425 & -0.088 & 0.060 & 0.041 & 22.149 & -0.007 & 0.000 & 0.023 & 21.472 & -0.091 & 0.000 & 0.020 \\
\hline 1999 Sep $26 \ldots \ldots$. & 22.204 & -0.047 & 0.060 & 0.037 & 22.030 & -0.006 & 0.000 & 0.040 & 21.342 & -0.100 & 0.000 & 0.071 \\
\hline 1999 Sep $27 \ldots \ldots$. & 22.208 & -0.147 & 0.060 & 0.016 & 22.045 & -0.050 & 0.000 & 0.014 & 21.363 & -0.108 & 0.000 & 0.031 \\
\hline 1999 Sep $28 \ldots \ldots \ldots$ & 22.211 & -0.130 & 0.060 & 0.030 & 22.054 & -0.064 & 0.000 & 0.019 & 21.392 & -0.105 & 0.000 & 0.064 \\
\hline 1999 Sep $29 \ldots \ldots$. & 22.240 & -0.125 & 0.060 & 0.028 & 22.072 & -0.073 & 0.000 & 0.021 & 21.381 & -0.110 & 0.000 & 0.026 \\
\hline
\end{tabular}

NoTE.-Photometric solutions are listed for all the clear nights. Col. (1) lists the UT date of the observations. Cols. (2)-(4), (6)-(8), and (10)-(12) list the coefficients of the photometric solutions defined in $\$ 3.2$. Cols. (5), (9), and (13) list the rms scatter in the solution for each filter and each night.

then flat-fielded and sky-subtracted using "running" flat and sky frames. The running flats were created by averaging the six neighboring images using a percentile clipping algorithm to remove bright objects. This image was then normalized to unity to create a sky flat for each image. The running sky frames were similarly created by averaging the six neighboring frames. At this stage the images were inspected, and images with anomalously high noise or serious tracking or guiding errors (generally two to three per field) were removed from further processing. The good images were then shifted and co-added together using an offset table of integer shifts to create a final mosaic image. This image was used to identify objects and, together with the offset table, was used to make an object mask for each of the individual frames. The flat-field and sky-subtraction steps were then repeated using these individual object masks.

TIFKAM has a noise pattern in the lower half of the array due to vibration of the mechanical cryocooler. This pattern is a few counts in amplitude and runs along rows in the form of a sine wave with a period 6 times the width of the array. Because the period is much longer than the array width and the amplitude is less than the sky noise, simply subtracting the average of each row after masking out objects removes most of this feature. After the cryocooler pattern was subtracted, the individual images were shifted and added into the final mosaic. This region was then trimmed to include only the area of the sky present in more than $83 \%$ of the individual frames. As the offsets were generally small and consistent, this step produced a final mosaic of relatively constant noise with minimal loss of field. Several of the fields in Table 1 were initially observed under nonphotometric conditions. Calibration images of these fields were obtained on photometric nights, and these images were reduced in the same manner as above. Three to six bright stellar objects were used to calibrate the fields observed under nonphotometric conditions.

\subsection{Standards}

The standard stars were processed in an identical manner to that outlined above, though with two exceptions. First, the cryocooler pattern was not subtracted from these frames. Tests of the images with artificial stars showed that this pattern did not affect the photometry of bright stars. Second, the five to six observations of each standard were photometered individually, rather than one measurement of a shifted and added frame. The standard stars were measured with PHOT using a 15" radius aperture and a mean sky measured in an annulus extending from $25^{\prime \prime}$ to $35^{\prime \prime}$ radius.

To convert the instrumental magnitudes to the CTIO CIT system, the following equations were solved:

$$
\begin{aligned}
J & =j+j_{0}+j_{1}(X-1.3)+j_{2}(J-K-1.4), \\
H & =h+h_{0}+h_{1}(X-1.3)+h_{2}(J-K-1.4), \\
K & =k+k_{0}+k_{1}(X-1.3)+k_{2}(J-K-1.4),
\end{aligned}
$$

where $j, h$, and $k$ are the instrumental magnitudes, $\mathrm{j}_{0}, h_{0}$, and $k_{0}$ are the photometric zero points, $j_{1}, h_{1}$, and $k_{1}$ are the air-mass coefficients, and $j_{2}, h_{2}$, and $k_{2}$ are the color terms. $X=1.3$ is the mean air mass of the fields observed in this survey, and $J-K=1.4$ is approximately the mean $J-K$ color of galaxies in the range $K=16-19$ reported by Saracco et al. (1999). While the photometric zero points and air-mass coefficients may vary from night to night, the color terms should not vary so long as the filters remain unchanged. To take advantage of the observations of red stars over several nights, I therefore performed a multidimensional fit (e.g., Gould 1995) to all the nights of a given observing run simultaneously. For each filter, this allowed the zero point and air-mass coefficient to vary but not the color term. Over a given observing run, the photometric zero point varied by a few percent. The air-mass coefficient was more variable, with large changes generally temporally correlated with changes in the weather (as discussed by 
Frogel 1998). If standards at large air mass were not observed on a particular night, the average coefficient of the temporally adjacent nights on that observing run was used. Most of the fields were observed at $X<1.5$ (and all at $<2$ ) so uncertainties in the air-mass coefficients translate to less than $1 \%$ uncertainties in the photometry. All the photometric solution coefficients are listed in Table 2, along with the rms variation in these solutions.

As mentioned above, there were several changes to the filters in TIFKAM over the course of this project. The $J$ filter used in 1997 October had considerable structure in the wings of the point-spread function (PSF), and therefore it was replaced before the 1998 April observing run. The new $J$ filter had a red leak such that the measured sky brightness was $\Sigma_{J} \approx 13 \mathrm{mag} \operatorname{arcsec}^{-2}$, compared with measurements of $\Sigma_{J} \approx 15.5-16 \mathrm{mag} \operatorname{arcsec}^{-2}$ on the other observing runs. After 1998 April this $J$ filter was used only in conjunction with a piece of PK50 glass in the prefilter wheel, which serves as a red-blocking filter. PK50 is opaque longward of $2.7 \mu \mathrm{m}$, but it is transparent at shorter wavelengths. A piece of PK 50 was also added to the same filter cell as the $H$ filter between the 1998 April and 1998 September observing runs because of the possible presence of a slight red leak in this filter. Because of the changes in the $J$ and $H$ filters, the photometric solution has three separate color terms for the $J$ filter and two separate color terms for the $H$ filter. The $K$ filter was unchanged over the course of this observing program and therefore only one color term was sought in this equation.

\subsection{Object Catalogs}

I cataloged the positions of all objects in these fields with the SExtractor package (Bertin \& Arnouts 1996) and the default object detection filter. This choice was motivated by the data reduction procedure, which creates images with "dead" regions, corresponding to areas with low exposure times and higher sky noise in the final shift-and-add step. The weight map option in SExtractor is an efficient way to insure detections on only the useful part of each image file. In addition, the noise varies somewhat over our images, and specifically the northern half of the array is on average noisier than the southern half. I compared SExtractor to FOCAS (Jarvis \& Tyson 1981; Valdes 1982) and found that SExtractor did a better job of detecting all the objects in the fields without introducing significant spurious detections.

\section{DETECTION EFFICIENCIES}

The limiting magnitude of a survey is typically expressed as the apparent magnitude for which the probability of detecting an object is equal to some percentage, commonly $50 \%$ or $90 \%$. To obtain an accurate census of all objects to such limiting magnitudes, one can use an estimate of the incompleteness versus apparent magnitude to correct the observed number of objects to the true value. This simple characterization of the detection efficiency is sufficient for the study of objects that all have the same size and surface brightness profile and is simplest to apply to point sources such as stars or quasars. I discuss the related issue of stargalaxy separation below in $\S 6$.

In studies of galaxies, more compact and higher surface brightness objects are generally easier to detect. Not taking the relative "visibility" (Disney 1976; Disney \& Phillipps 1983; Phillipps, Davies, \& Disney 1990; Davies 1990) of different types of objects into account can lead to erroneous conclusions about the intrinsic distribution of observed galaxy properties. The detection efficiency of a survey should therefore be characterized as not just a function of apparent magnitude but also as a function of surface brightness profile and angular size. That is, surveys should take into account the multivariate nature of the galaxy population rather than collapsing variations in profile shape, size, and surface brightness into just a dependence on integrated brightness. Bershady et al. (1998), for example, separate objects into stars and small and large galaxies and calculate the detection efficiency of each class separately.

The surface brightness profile of galaxies is commonly parameterized by either an exponential profile,

$$
\Sigma(r)=\exp \left(-1.673 \frac{r}{r_{h}}\right),
$$

or a de Vaucouleurs $r^{1 / 4}$ profile,

$$
\Sigma(r)=\exp \left\{-7.67\left[\left(\frac{r}{r_{h}}\right)^{1 / 4}-1\right]\right\},
$$

where $\Sigma(r)$ is the surface brightness at radius $r$ and $r_{h}$ is the scale radius for each profile that encloses half the total light. Most galaxies can be fitted by either one of these profiles or, for disk galaxies with a bulge component, a superposition of both. These parameterizations are useful for examining how the detection efficiency varies as a function of galaxy size and profile shape.

The sensitivity of each of the fields in this survey is different because of variations in the atmospheric conditions and instrument setup. The likelihood of detecting an object of a given brightness, size, and profile varies from field to field and cannot be accurately represented by a single number for the entire survey, even for stellar objects. To characterize the detection limits of this survey, I added artificial objects produced with the IRAF ARTDATA package to each of the fields in each filter and measured the fraction of recovered objects with SExtractor. In addition to stellar profiles, I added galaxies with both exponential and $r^{1 / 4}$ profiles with $r_{h}$ equal to $0.25,0.5,0$.".75, and $1^{\prime \prime}$ over a range of apparent magnitude. Studies of the HST Medium Deep Survey (Roche et al. 1997), the NICMOS parallel survey (Teplitz et al. 1998; Yan et al. 1998), and HST imaging of the CFRS and LDSS galaxies (Lilly et al. 1998) have shown that to the apparent magnitude limits of this survey nearly all galaxies are well fitted by either exponential or $r^{1 / 4}$ profiles and most have $r_{h}<1^{\prime \prime}$ and exponential profiles. An alternative to using artificial objects to measure the detection efficiency is to extract bright objects from the survey fields, artificially dim them, place them at random back into the original images, and attempt to recover them. However, as the median galaxy size declines at fainter apparent magnitudes, this may lead to an underestimate of the detection efficiency.

I convolved the model galaxy profiles used in this analysis with a Moffat (1969) profile as this profile is a much better fit to the observed profiles of bright stars than a Gaussian profile. A Moffat profile has the functional form

$$
I(r)=\left[1+(r / \alpha)^{2}\right]^{\beta} .
$$

After fitting Moffat profiles to bright stars in a number of fields and filters that spanned the range of PSF sizes, I fixed $\beta$ to be 2.5. The parameter $\beta$ determines the strength of the 
power-law tail of the radial intensity distribution, while $\alpha$ parameterizes the width of the profile peak and is similar to $\sigma$ in a Gaussian function. This procedure set the convolution kernel for the model galaxy profiles in ARTDATA for each image and takes the variation in PSF from image to image into account in the determination of the detection efficiency. The functional form of the Moffat profile used by the ARTDATA package is slightly different from equation (4), but MOFFAT profiles produced by ARTDATA are in reasonable agreement with this standard form. The default dynamic range of the convolution kernel in ARTDATA for galaxy profiles (ARTDATA.DYNRANG) is only 10, which causes an artificial truncation in galaxy profiles. I changed the value of this parameter to 10,000 , which matches the dynamic range of the PSF computation. While this is more computationally intensive, it is not prohibitive.

In Figure 1, I show the relative detection efficiency for these two profiles at range $r_{h}$ in a field of fixed sky brightness. The sky noise and magnitude zero point in this figure are from one of the $K$ band frames, although the relative detection efficiencies for different sizes, profiles, and seeing are independent of the actual filter used. Throughout this paper I will use $r_{h}$ to parameterize the angular half-light radius rather than associate it with a physical scale. The top two panels of Figure 1 show an exponential disk model, and the bottom two panels show an $r_{h}$ profile. The left panels correspond to a seeing FWHM of 1", and the right panels correspond to 1 ".5 seeing. This figure shows that the detection efficiency for stellar objects is $0.5-1 \mathrm{mag}$ fainter than the detection efficiency for marginally resolved galaxies and that the detection efficiency is similar for exponential disks and $r^{1 / 4}$ profiles with the same $r_{h}$. As angular size and surface brightness vary with redshift, the detection efficiency for different classes of objects can be even more important for selecting galaxies for a redshift survey that will be used to compute the luminosity function (Dalcanton 1998a; Petrosian 1998).

Figure 2 illustrates the variation in detection efficiency from field to field of this survey as a function of object size. The histogram represents the number of square arcminutes with a given $90 \%$ completeness limit versus $K$ magnitude for the $185 \mathrm{arcmin}^{2}$ of this survey. The top panel shows the

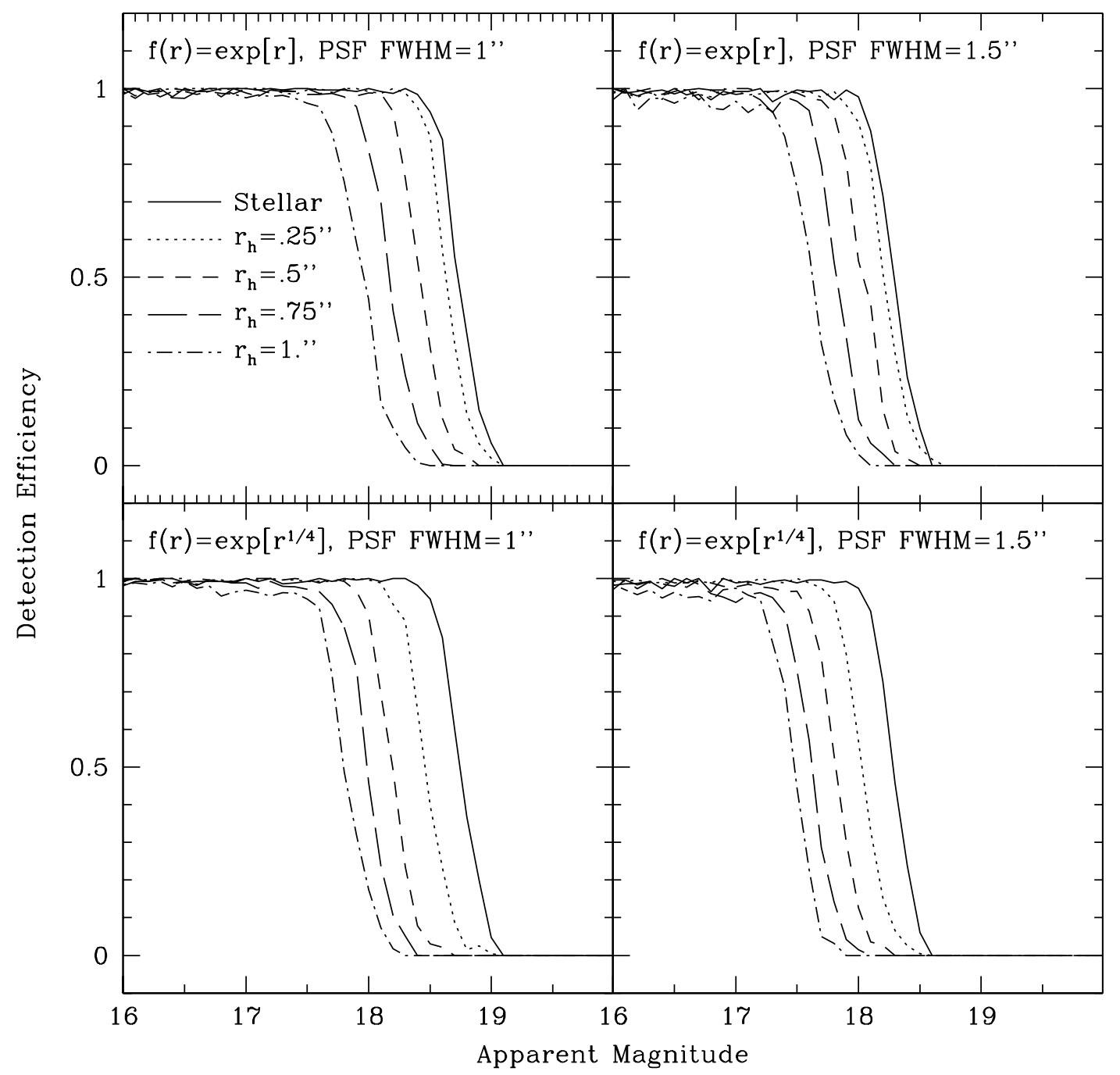

FIG. 1.-Variation in the detection efficiency vs. apparent magnitude and source size. Top left: Detection efficiency vs. apparent magnitude for a stellar profile (PSF FWHM $=1^{\prime \prime}$ and Moffat $\beta=2.5$ ) and four exponential disks with intrinsic half-light radii of $r_{h}=00^{\prime \prime} .25,0^{\prime \prime} .5,0^{\prime \prime} .75$, and $1^{\prime \prime}$. The exponential disks have all been convolved with the 1" PSF. Bottom left: Same as top left except that the intrinsic galaxy surface brightness profile is a de Vaucouleur model. Top right and bottom right: Graphs from the same image, except with PSF FWHM =1".5. The sky noise and magnitude zero point in this figure are from one of the $K$ band frames, although the relative detection efficiencies for different sizes, profiles, and seeing are independent of the actual filter used. 


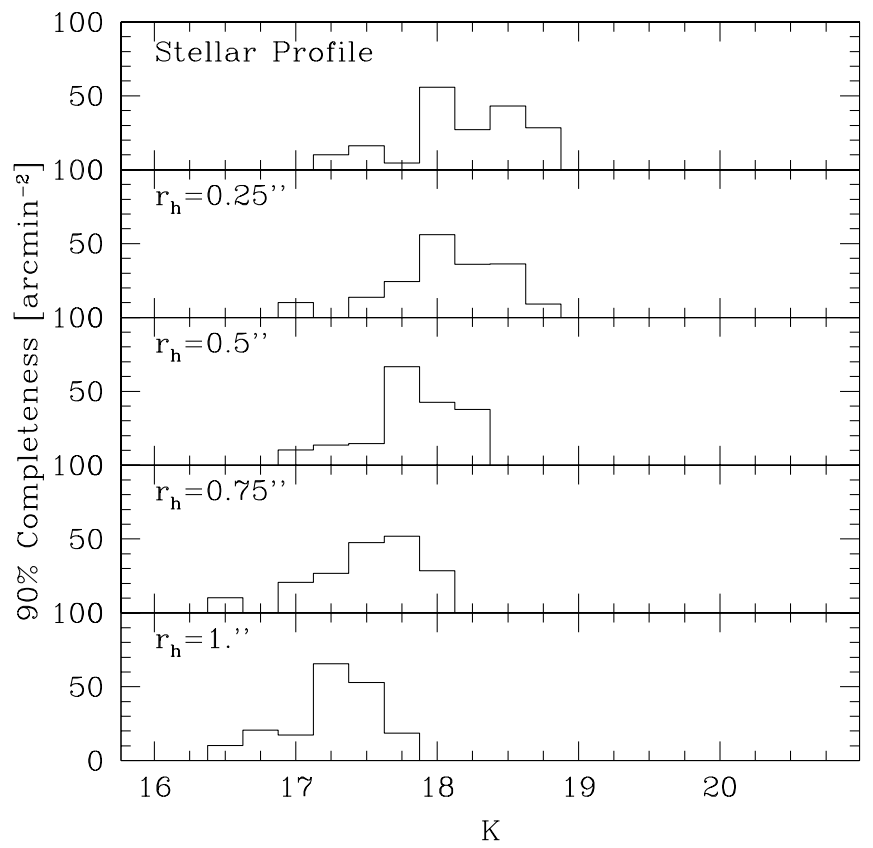

FIG. 2.-Histogram of survey area with a given $90 \%$ limiting $K$ magnitude. Each 0.25 mag bin shows the number of square arcminutes that are $90 \%$ complete to that $K$ apparent magnitude for the given object size. Top, distribution for stellar profiles; middle and bottom, distribution for exponential disks with half-light radii of $r_{h}=0$ "'.25, 0 ".5, 0"75, and $1^{\prime \prime}$.

distribution for stellar profiles, while the lower panels show exponential disks with $r_{h}=00^{\prime \prime} 25,0$. 5,0 , .75 , and $1^{\prime \prime}$. The average area-weighted $50 \%$ completeness limits of the survey are $J=20.5, H=19.5$, and $K=18 \mathrm{mag}$ for exponential disks with $r_{h}=0.75$. For stellar objects these limits are $J=21, H=20$, and $K=18.5 \mathrm{mag}$.

\section{PHOTOMETRY}

The unknown intrinsic morphology of galaxies makes galaxy photometry considerably more challenging than measuring the apparent magnitude of a star. In this paper, the goal is to measure the total, integrated brightness of galaxies to compare with models of galaxy surface density as a function of integrated brightness. Most of the galaxies in this sample have small angular sizes, where "small" in this context means $r_{h}$ less than or equal to the seeing FWHM. Because of the finite signal-to-noise ratio and the lack of numerous bright stars in each field to determine exactly the PSF, the true morphology of the galaxies cannot be accurately deconvolved. Photometric techniques for marginally resolved galaxies include aperture magnitudes, isophotal magnitudes, metric magnitudes, and various slight modifications of these techniques.

Aperture magnitudes are the simplest to define as they just involve computing the total flux within some given size aperture. The problem with aperture magnitudes, however, is that they always exclude some fraction of a galaxy's light unless the aperture is made very large. While using a very large aperture is a way of avoiding systematic errors in galaxy photometry, it is impractical to apply to observations within several magnitudes of the survey limit because of nonnegligible sky noise. A pragmatic approach is therefore to measure the brightness of the galaxy within some small aperture and then to correct this aperture magnitude to compensate for the lost light; the correction is usually based on some measure of the galaxy size (Glazebrook et al. 1994; Soifer et al. 1994; Cowie et al. 1994; Bershady et al. 1998; Saracco et al. 1999). For any size aperture, the fraction of lost light depends on both the scale size and surface brightness profile of the galaxy. These quantities are difficult to measure with great accuracy for galaxies near their detection limit.

An isophotal magnitude is measured by summing all a galaxy's flux out to some limiting isophote, usually the limiting isophotal magnitude of the survey. A variation of the isophotal magnitude called the "TOTAL" magnitude is more commonly used (McLeod et al. 1995; Hall, Green, \& Cohen 1998; Minezaki et al. 1998b) and is one of the quantities calculated by FOCAS. The FOCAS TOTAL magnitude is defined as the sum of the flux within twice the isophotal area, rather than just the isophotal area. The isophotal magnitude is well known to be biased toward underestimating the flux of all but very bright and high surface brightness galaxies. Using the FOCAS TOTAL magnitude decreases the tendency of isophotal magnitudes to underestimate galaxy size and integrated brightness by effectively increasing the aperture size, but it is fundamentally still an isophotal magnitude and therefore underestimates the integrated light of galaxies (Dalcanton 1998b).

Metric magnitudes appear to offer a physically motivated solution to the shortcomings of these two techniques. The most common metric magnitude is measured within the radius introduced by Petrosian (1976). The Petrosian magnitude is defined to be the flux interior to radius $r_{p}$, where the surface brightness at $r_{p}$ is equal to some fraction of the average surface brightness interior to $r_{p}$. The great attraction of using a Petrosian magnitude is that it is independent of the spacetime geometry by virtue of being the ratio of two surface brightnesses. Therefore $r_{p}$ corresponds to the same physical scale in two galaxies with the same surface brightness profile at different redshifts. The corresponding integrated brightness within that radius corresponds to the same fraction of the total light (any systematic underestimate will be identical for the two galaxies). However, the fraction of the light missed by a Petrosian magnitude for two galaxies at different redshifts is the same only if two conditions hold: the surface brightness profiles of the galaxies are the same and neither of the galaxies is small enough to be significantly affected by the PSF. Thus while the Petrosian magnitude is ideal for cosmological tests (Sandage \& Perelmuter 1990; Petrosian 1998), it may not offer the ideal choice for measuring the integrated light of galaxies with a diverse range of surface brightness profiles and sizes comparable to the PSF of the observations.

The main goal of the magnitude measurements in this paper is to measure the number of galaxies per unit magnitude. These measurements, in conjunction with theoretical predictions, can then be used to constrain models of galaxy formation and evolution. The output of the theoretical models with which observed number counts are compared is the sum of galaxies in a given apparent magnitude bin. To compare observational data with these theoretical predictions therefore requires an unbiased measurement of the number of galaxies at a given integrated brightness, which in turn requires a good means of estimating the integrated brightness of individual galaxies.

I evaluated different techniques for measuring the integrated brightness of the galaxies in the sample, including aperture magnitudes measured with PHOT in IRAF, 
TOTAL magnitudes measured with FOCAS, and "BEST" magnitudes measured by SExtractor. The SExtractor BEST magnitude is defined to be either a Kron magnitude (Kron 1980), which is measured in an elliptical aperture whose size is determined by the object's profile, or an isophotal magnitude in very crowded regions. To evaluate the accuracy of these photometry techniques I used ARTDATA in IRAF (as described in $\S 4$ ) to add artificial galaxies to the images. The metric to evaluate the accuracy of each of these techniques is the size of the difference between the input magnitude and the measured magnitude as a function of $r_{h}$ for exponential and $r^{1 / 4}$ profiles. I performed these tests for different images to sample the range of seeing in the data and at a range in apparent magnitude from the 50\% completeness limit for stellar profiles to 4 mag brighter. This magnitude range includes more than $80 \%$ of the sources in the sample. The investigation showed that IRAF PHOT aperture magnitudes, FOCAS TOTAL magnitudes, and SExtractor BEST magnitudes all underestimate the integrated brightness of galaxies. The FOCAS and SExtractor magnitudes were taken directly from the output of these two packages, while I added a stellar profile aperture correction based on measurements of several stellar objects in the field to the aperture magnitudes measured by IRAF PHOT.

Figure 3 shows the result of one of these experiments for an image with 1".25 FWHM. The measured magnitude is the IRAF PHOT aperture magnitude (left), FOCAS TOTAL magnitude (middle), or SExtractor BEST magnitude (right), and the true magnitude is the input magnitude assigned to the artificial object. As in Figure 1, the sky noise and apparent magnitude for this simulation are based on one of the $K$ band frames; however, the reliability of these different photometric techniques relative to the completeness limit is independent of the filter. This figure shows the results for stellar profiles (top, left to right), $r_{h}=0$ ".5 exponential disks (middle, left to right), and $r_{h}=1^{\prime \prime}$ exponential disks (bottom, left to right). The data points are the mean offset between the measured and true magnitudes from $\sim 200$ artificial objects per apparent magnitude, and the

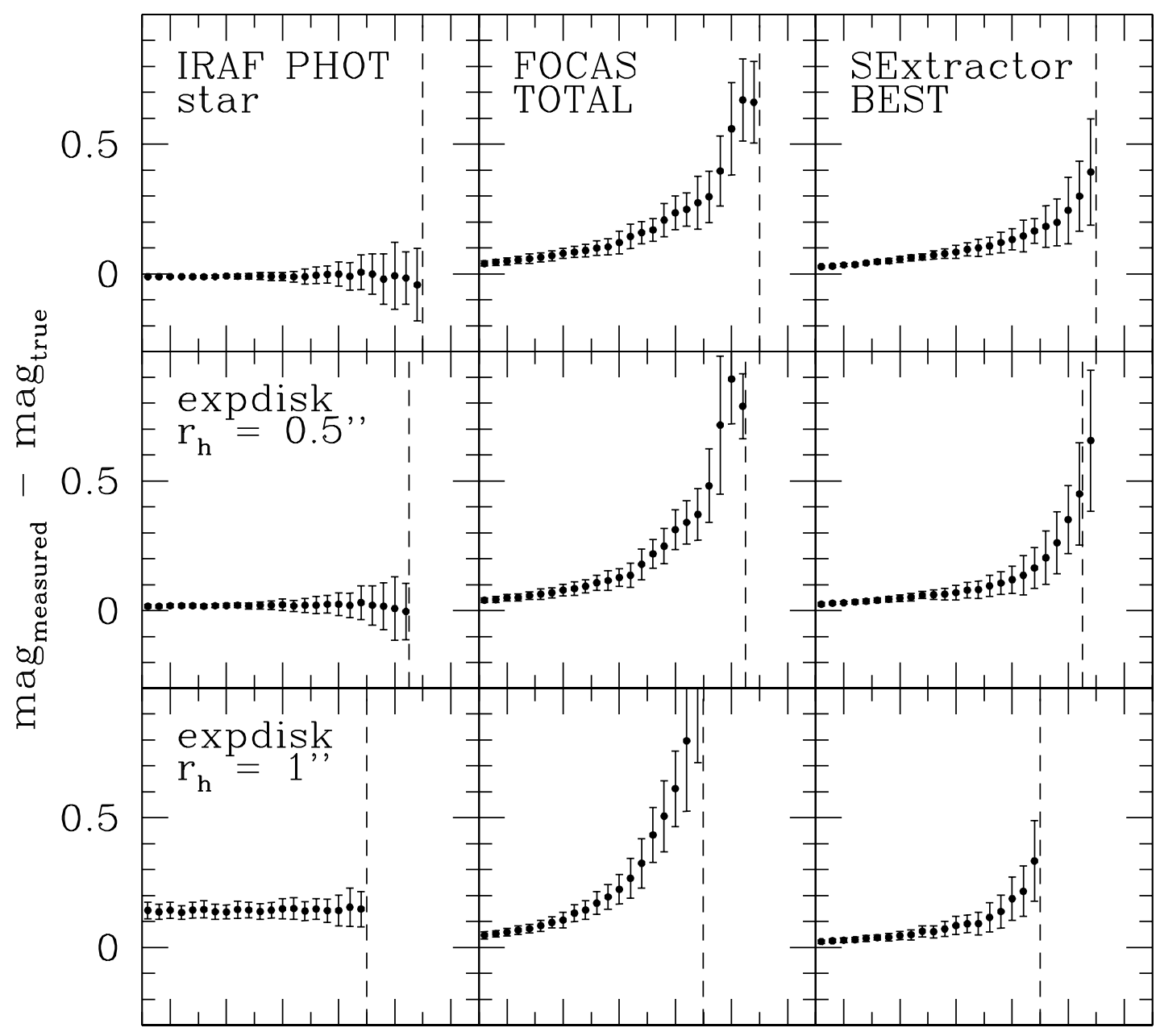

151617181920151617181920151617181920

Apparent Magnitude

FIG. 3.-Reliability of the photometric methods as a function of image size and integrated brightness. Left, difference between the measured and true magnitudes for aperture photometry with IRAF PHOT; middle, same but with FOCAS TOTAL magnitudes; right, same but with SExtractor BEST magnitudes; top to bottom, results for stellar objects, exponential disks with $r_{h}=0.5$, and exponential disks with $r_{h}=1^{\prime \prime}$, respectively. The dashed vertical line is the $90 \%$ detection limit for the objects in each panel. As in Fig. 1, the sky noise and magnitude zero point were taken from one of the $K$ band frames; however, these results depend only on the apparent magnitude relative to the magnitude of the $90 \%$ detection limit. 
error bars are the $1 \sigma$ dispersion in these measurements at that magnitude. At a fixed input galaxy brightness more light is missed in galaxies with larger $r_{h}$, while at fixed $r_{h}$, more light is missed in an $r^{1 / 4}$ profile than in an exponential profile. For fainter apparent magnitudes, the size of the brightness underestimate increases for the FOCAS TOTAL and SExtractor BEST magnitudes at fixed $r_{h}$, but it remained constant for the aperture magnitude. The reason for the systematic increase in the underestimate for the FOCAS TOTAL magnitudes is that the isophotal area of a galaxy of fixed size decreases as the integrated brightness decreases. Though the FOCAS TOTAL magnitude doubles the isophotal area, this still leads to an underestimate of the true size of the galaxy for the photometric measurement. The SExtractor BEST magnitude suffers from the same systematic increase in error as the size of the elliptical aperture is set by a fit to the observed galaxy profile. This offset is also due to the fact that SExtractor BEST magnitudes assume the PSF is Gaussian when computing the elliptical aperture for photometry and that a Gaussian profile is more centrally concentrated than a MOFFAT profile (Dalcanton 1998b). At a higher galaxy surface density than probed in this survey, the SExtractor BEST magnitudes are isophotal magnitudes rather than Kron magnitudes, and the brightness underestimate may be more severe. For fainter galaxies at fixed size and profile shape a smaller fraction is above the noise, and the tendency is for a detected object to appear more compact, which further shrinks the aperture size used in the photometry. While I have not evaluated Petrosian magnitudes here, Dalcanton (1998a) included them in her study of systematic biases in measuring the integrated light of galaxies for computing the luminosity function. She found that Petrosian magnitudes underestimate the true brightness of galaxies to a lesser extent than aperture or isophotal magnitudes do (see also Dalcanton 1998b), although they too are subject to large errors for objects near the detection limit. This is because the surface brightness profile becomes increasingly noisy. In addition, as stated above, the Petrosian radius is difficult to determine for marginally resolved objects.

Saracco et al. (1999) also compare aperture magnitudes with isophotal magnitudes and SExtractor AUTO magnitudes, which are similar to SExtractor BEST magnitudes. Their Figure 1 shows a difference of several tenths of a magnitude between photometric measurements with these three techniques of the galaxies in their fields. These differences increase to on order $1 \mathrm{mag}$ for the last 2 mag below their $S / N=5$ detection limit for compact objects. They find that both isophotal and SExtractor AUTO magnitudes underestimate the flux of faint objects and therefore chose to use aperture magnitudes. Väisänen et al. (2000) have also evaluated the relative merits of aperture, isophotal, and SExtractor BEST magnitudes. They concluded that SExtractor BEST magnitudes do not underestimate the integrated brightness of galaxies near the detection limit, in contrast with the simulations presented here and the results of Dalcanton (1998b).

My analysis has shown that only aperture magnitudes avoid errors in the photometry that are a function of integrated brightness, and I have chosen to use them. The brightness underestimate of aperture photometry still depends on the intrinsic size and profile shape, but it avoids the additional systematic uncertainty due to the dependence on integrated brightness inherent in SExtractor BEST and
FOCAS TOTAL magnitudes. Another consideration in the decision to use aperture magnitudes is the unique nature of these data. I have 21 fields observed in three filters, each with a different PSF shape. Aperture photometry is the best way to account for the heterogeneous nature of the data set. The size of the error in the SExtractor BEST and FOCAS TOTAL magnitudes varies from field to field with the seeing. While aperture magnitudes suffer from the same problem, they can be relatively easily scaled to take seeing variations into account. In addition, the aperture magnitudes can be easily measured through a sufficiently large aperture to minimize the size of the galaxy aperture correction and therefore the associated uncertainty.

Given the range of galaxy sizes and typical seeing, aperture magnitudes with a radius 1.5 times the FWHM of the seeing are a good compromise between minimizing the size of the galaxy aperture correction and maximizing the galaxy signal in the aperture. At $z=0.5,1^{\prime \prime}$ corresponds to a physical size of $4.3 h^{-1} \mathrm{kpc}$ (for $\Omega_{M}=0.3$ and $\Omega_{\Lambda}=0.7$ ), and therefore these typical aperture sizes of 1".5-2".5 include most of the galaxies' light. The galaxy aperture correction is the difference between the true integrated brightness of the galaxy and the measured aperture magnitude plus the stellar aperture correction. The stellar aperture correction depends only on the shape of the PSF, and, because the aperture is scaled to the PSF size, this correction was always measured to be $\sim 0.1 \mathrm{mag}$. The galaxy aperture correction depends on the typical galaxy size and was measured with the same code developed to measure the detection efficiency for objects of different size, surface brightness profile, and integrated brightness. The distributions of size and surface brightness profile as a function of integrated brightness have not been completely determined in the NIR. However, a range of studies of galaxy morphology at visible (Smail et al. 1995; Roche et al. 1997, 1998) and NIR (Yan et al. 1998; Bershady et al. 1998; Saracco et al. 1999) wavelengths, combined with color information (Thompson et al. 1999), shows that most of the faint galaxies in this survey have exponential profiles with typical half-light radii of 0 ".5-0".75. At the magnitude limits of this survey the average galaxy size is $r_{h} \sim 0$ ". 6 in these three filters. Any bulge component for these galaxies, though more prominent at NIR wavelengths, falls inside the aperture. For this range of sizes of galaxies, I computed the galaxy aperture correction for each frame as a function of integrated brightness. The size of this correction is always in the range $0.1-0.2 \mathrm{mag}$. The correction is generally smaller for fields with poorer seeing as the aperture size is larger in these fields, although the detection limit for a given galaxy size and profile shape is lower for these fields as well.

\section{STAR-GALAXY SEPARATION}

The calculation of number counts of galaxies at faint magnitudes must deal with stellar contamination, which affects faint galaxy surveys in two ways. First, the typical galaxy size decreases at fainter apparent magnitudes, and therefore galaxies are progressively more likely to be morphologically indistinguishable from stars. Second, stars (and compact galaxies) are the easiest objects to detect and accurately photometer near the survey limits. This is illustrated in Figure 1, which shows that the limiting magnitude at fixed detection efficiency is on order $1 \mathrm{mag}$ brighter for exponential disks or $r^{1 / 4}$ profiles with $r_{h}=1^{\prime \prime}$ than for stars. Multicolor separation is one way to remove stellar contami- 
nation from faint galaxy samples. This process is most effective with small photometric errors, although it can be effective with larger errors with a longer wavelength baseline (e.g., visible to NIR colors; Gardner 1995). The removal of stellar contamination from this survey is particularly important as stars contribute on order $20 \%-30 \%$ to even the faintest magnitude bins of this survey. In even deeper surveys stellar contamination is less of a problem as galaxies begin to significantly outnumber stars.

The stellar content of the DMS fields has already been cataloged as part of the search for quasars (Hall et al. 1996a; Osmer et al. 1998). The selection criterion for identifying stellar objects was that they must be indistinguishable from the PSF in at least three of the six CCD filters (Hall et al. 1996a). I used the stellar catalog from Osmer et al. (1998) to identify and filter out stars from the NIR catalogs. Given that the CCD stellar catalog extends several magnitudes fainter than this survey, all but the very reddest stars should be included in the stellar catalog. In contrast, the CCD stellar catalog includes some contamination from compact galaxies near its magnitude limit. From the stellar model counts presented in Minezaki et al. (1998a) and galaxy counts in the literature, I expect stars compose approximately $10 \%-20 \%$ of all objects at the limiting magnitude of this survey. Any missed fraction will therefore be a minor contamination in the galaxy number counts.

\section{NUMBER COUNTS}

The calculation of NIR galaxy number counts in the DMS is complicated by the variation in detection efficiency from field to field. To combine all the fields I have used a technique analogous to the one outlined in the appendix of Bershady et al. (1998). However, the same variations in seeing that contribute to the different field sensitivities make it difficult to bin these objects reliably into different size classes. I have therefore not separated the counts into different sizes (and profile types), effectively collapsing the galaxy distribution from a multivariate distribution (size, profile shape, and integrated brightness) to a function of integrated brightness alone. As most number-magnitude models for galaxies predict only surface density versus integrated brightness (though see Im et al. 1995), this is the most useful format to compare the data with theoretical predictions.

For each apparent magnitude bin I used the typical galaxy size and galaxy aperture correction discussed in $\S 5$ to correct the measured brightness of each galaxy. All the galaxies in each field were then placed into $0.5 \mathrm{mag}$ bins in apparent magnitude. The detection efficiency for each bin is the average of the detection efficiency versus magnitude value measured in $0.1 \mathrm{mag}$ increments and weighted by the expected slope of the number-magnitude relation $\alpha \sim 0.5$, where $\log N \propto \alpha m$. In practice, the detection efficiency drops off significantly faster than the number-magnitude relation increases, and so the detection efficiency of a magnitude bin is relatively insensitive to $\alpha$. If the detection efficiency of the faint end of a bin was less than $50 \%$, this bin was not included in the calculation of the number-magnitude relation. The detection efficiencies described in $\S 4$ multiplied by the field size yields the effective area of each field. I then summed the total differential counts and effective area of each field per $0.5 \mathrm{mag}$ bin to compute the differential number counts per unit magnitude per square degree. The number counts for $J, H$, and $K$ are shown in Figures 4, 5 , and 6 , respectively. The raw and corrected counts, along

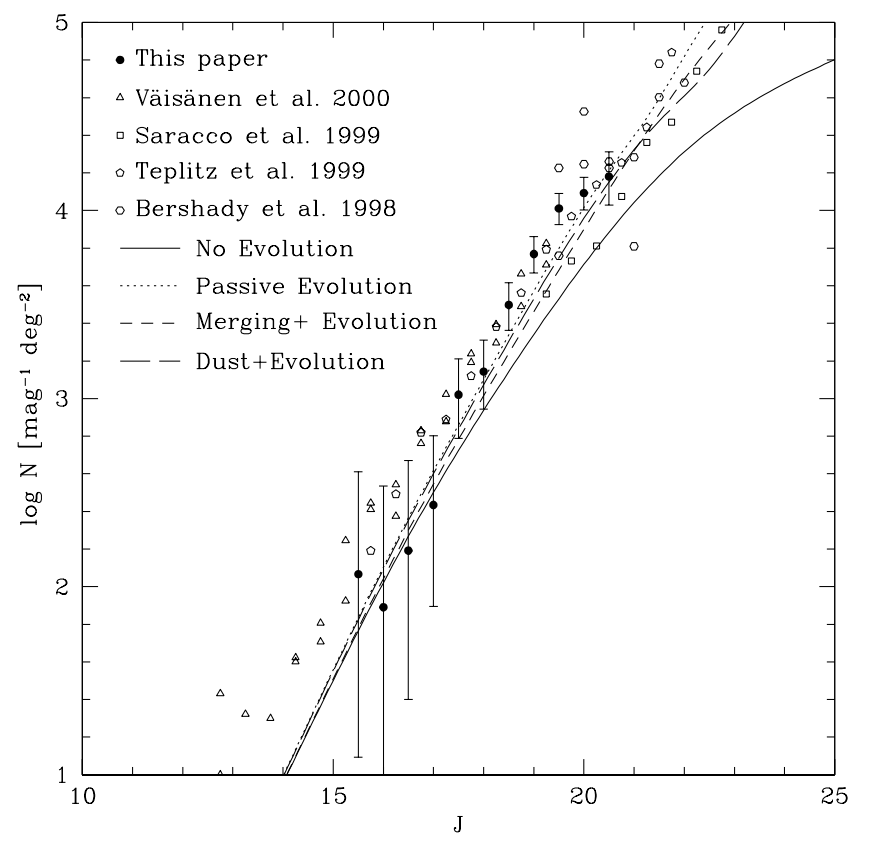

FIG. 4.-Differential galaxy number counts in the near-infrared $J$ band. The raw number counts have been converted to units of $\mathrm{mag}^{-1} \mathrm{deg}^{-2}$ (filled circles) and the error bars are $1 \sigma$ confidence limits. These data are listed in Table 3. Also shown are number counts from the literature and four models from Gardner (1998).

with the effective area, are listed in Tables 3,4 , and 5 . The raw counts are the total counts in 0.5 mag bins with only the stellar $(\sim 0.1 \mathrm{mag})$ aperture correction applied to the photometry. The corrected counts are the counts per 0.5 mag bin after application of the galaxy aperture correction. The error bars on the number counts are $1 \sigma$ upper and lower confidence intervals calculated using the formulae in Gehrels (1986).

The $J$ number counts shown in Figure 4 agree well with the number counts of Teplitz et al. (1999), Väisänen et al.

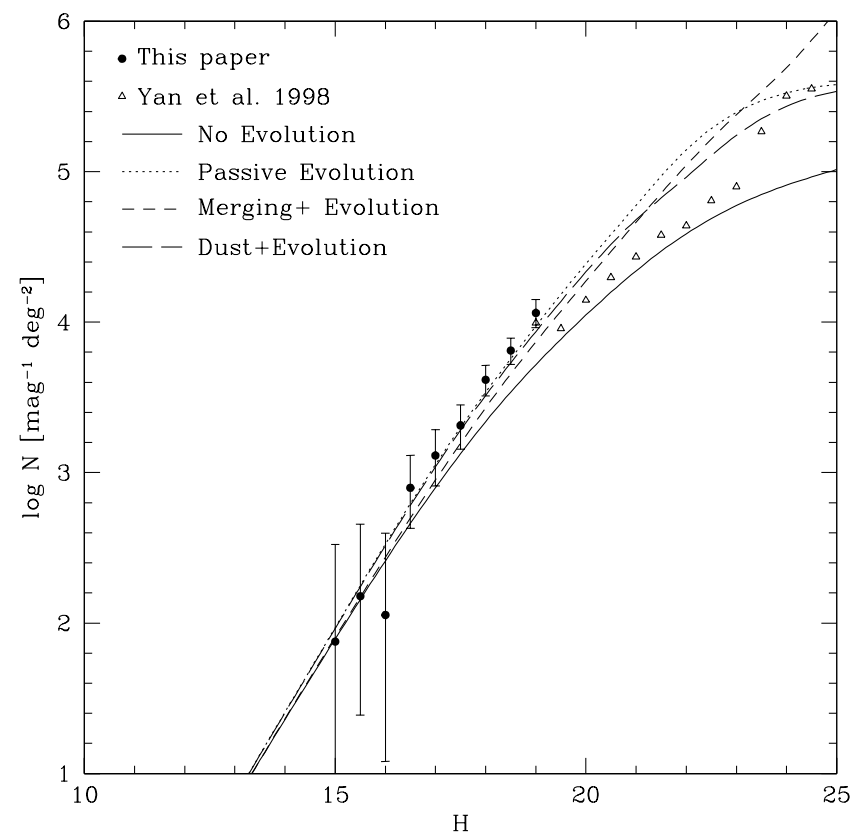

Fig. 5.-Differential galaxy number counts as in Fig. 4 but for the near-infrared $H$ band. These data are listed in Table 4. 


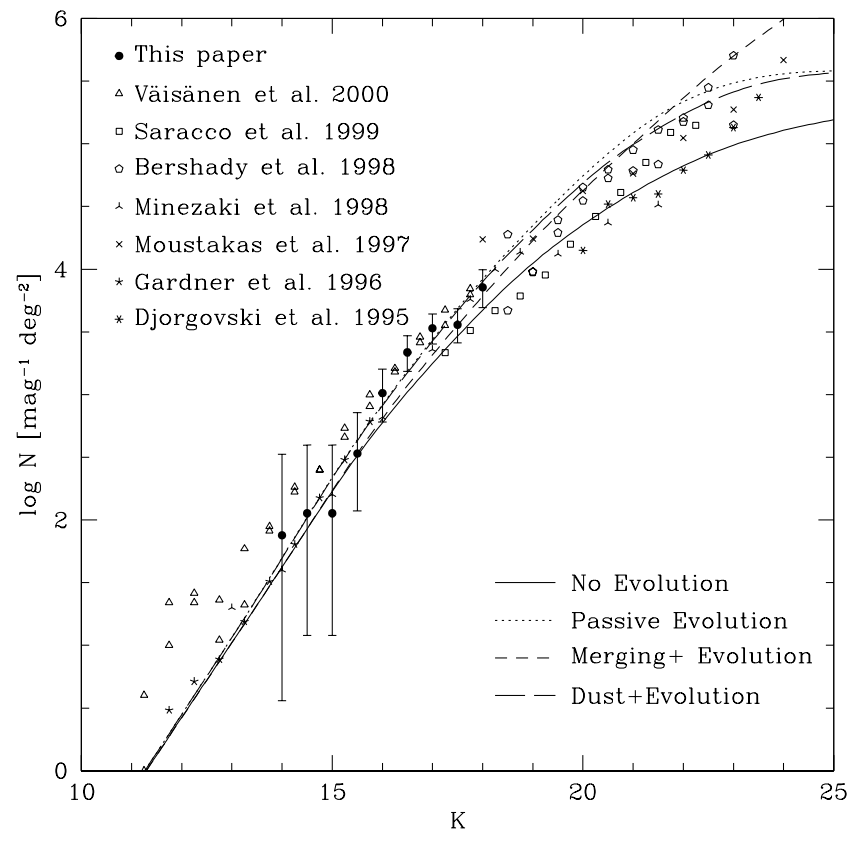

Fig. 6.-Differential galaxy number counts as in Fig. 4 but for the near-infrared $K$ band. These data are listed in Table 5 .

TABLE 3

DifFerential $J$ Number COUNTS

\begin{tabular}{crcrrr}
\hline \hline $\begin{array}{c}\text { Mag } \\
(1)\end{array}$ & $\begin{array}{c}N_{\text {raw }} \\
(2)\end{array}$ & $\begin{array}{c}\text { Area } \\
(3)\end{array}$ & $\begin{array}{c}N_{\text {corr }} \\
(4)\end{array}$ & $\begin{array}{c}N_{\text {low }} \\
(5)\end{array}$ & \multicolumn{1}{c}{$\begin{array}{c}N_{\text {upp }} \\
(6)\end{array}$} \\
\hline $15.50 \ldots \ldots$ & 2 & 0.05150 & 117 & 53 & 230 \\
$16.00 \ldots \ldots$ & 2 & 0.05150 & 78 & 28 & 180 \\
$16.50 \ldots \ldots$ & 4 & 0.05150 & 155 & 81 & 278 \\
$17.00 \ldots \ldots$. & 3 & 0.05150 & 272 & 172 & 418 \\
$17.50 \ldots \ldots$ & 28 & 0.05150 & 1049 & 848 & 1292 \\
$18.00 \ldots \ldots$. & 29 & 0.05037 & 1390 & 1156 & 1666 \\
$18.50 \ldots \ldots$ & 64 & 0.04453 & 3144 & 2769 & 3566 \\
$19.00 \ldots \ldots$. & 114 & 0.04022 & 5868 & 5329 & 6460 \\
$19.50 \ldots \ldots$ & 145 & 0.03041 & 10261 & 9440 & 11150 \\
$20.00 \ldots \ldots$. & 138 & 0.02356 & 12394 & 11370 & 13507 \\
$20.50 \ldots \ldots$. & 59 & 0.00753 & 15137 & 13139 & 17419 \\
\hline
\end{tabular}

NoTE.-Differential $J$ number counts of galaxies. Col. (1) lists the center of each $0.5 \mathrm{mag}$ bin. Col. (2) lists the raw number of galaxies detected in that magnitude range. Col. (3) lists the total effective area of that magnitude range in square degrees. Col. (4) contains the number of galaxies per magnitude per square degree after accounting for the aperture correction and detection probability. Cols. (5)-(6) show the $1 \sigma$ lower and upper confidence limits, respectively, computed as in Gehrels 1986.

TABLE 4

DifFERENTIAL $H$ NUMBER COUNTS

\begin{tabular}{crcrrr}
\hline \hline $\begin{array}{c}\text { Mag } \\
(1)\end{array}$ & $\begin{array}{c}N_{\text {raw }} \\
(2)\end{array}$ & $\begin{array}{c}\text { Area } \\
(3)\end{array}$ & $\begin{array}{c}N_{\text {corr }} \\
(4)\end{array}$ & \multicolumn{1}{c}{$\begin{array}{c}N_{\text {low }} \\
(5)\end{array}$} & \multicolumn{1}{c}{$\begin{array}{c}N_{\text {upp }} \\
(6)\end{array}$} \\
\hline $15.00 \ldots \ldots$ & 2 & 0.05310 & 75 & 27 & 175 \\
$15.50 \ldots \ldots$. & 4 & 0.05310 & 151 & 79 & 270 \\
$16.00 \ldots \ldots$ & 3 & 0.05310 & 113 & 52 & 223 \\
$16.50 \ldots \ldots$ & 12 & 0.05310 & 791 & 620 & 1004 \\
$17.00 \ldots \ldots$ & 28 & 0.05226 & 1301 & 1079 & 1565 \\
$17.50 \ldots \ldots$ & 59 & 0.05145 & 2060 & 1778 & 2384 \\
$18.00 \ldots \ldots$ & 97 & 0.05132 & 4131 & 3730 & 4572 \\
$18.50 \ldots \ldots$ & 130 & 0.04363 & 6463 & 5919 & 7054 \\
$19.00 \ldots \ldots$. & 122 & 0.02187 & 11524 & 10499 & 12644 \\
\hline
\end{tabular}

Note.-Same as Table 3 but for the differential $H$ number counts.
TABLE 5

DiFFERENTIAL $K$ NUMBER COUNTS

\begin{tabular}{cccrrr}
\hline \hline $\begin{array}{c}\text { Mag } \\
(1)\end{array}$ & $\begin{array}{c}N_{\text {raw }} \\
(2)\end{array}$ & $\begin{array}{c}\text { Area } \\
(3)\end{array}$ & \multicolumn{1}{c}{$\begin{array}{c}N_{\text {corr }} \\
(4)\end{array}$} & \multicolumn{1}{c}{$\begin{array}{c}N_{\text {low }} \\
(5)\end{array}$} & $\begin{array}{c}N_{\text {upp }} \\
(6)\end{array}$ \\
\hline $14.00 \ldots \ldots$ & 2 & 0.05310 & 75 & 27 & 175 \\
$14.50 \ldots \ldots$ & 3 & 0.05310 & 113 & 52 & 223 \\
$15.00 \ldots \ldots$ & 4 & 0.05310 & 113 & 52 & 223 \\
$15.50 \ldots \ldots$ & 5 & 0.05310 & 339 & 228 & 494 \\
$16.00 \ldots \ldots$ & 21 & 0.05248 & 1029 & 832 & 1268 \\
$16.50 \ldots \ldots$ & 50 & 0.05163 & 2169 & 1880 & 2499 \\
$17.00 \ldots \ldots$ & 59 & 0.04617 & 3379 & 2997 & 3807 \\
$17.50 \ldots \ldots$ & 81 & 0.03438 & 3607 & 3150 & 4126 \\
$18.00 \ldots \ldots$ & 50 & 0.01424 & 7163 & 6163 & 8312 \\
\hline
\end{tabular}

Note.-Same as Table 3 but for the differential $K$ number counts.

(2000), and the (small + large) counts of Bershady et al. (1998), though they are somewhat higher than the Saracco et al. (1999) counts. The Väisänen et al. (2000) survey overlaps the most with this survey, although they use SExtractor BEST magnitudes rather than aperture magnitudes. Their survey covers a substantially larger area than this one, though its faint limit is nearly 1 mag brighter. Bershady et al. (1998) and Saracco et al. (1999) extend much fainter than this survey, and their brightest counts overlap only the faintest magnitudes of this survey. In this regime they have approximately 5 to 10 times fewer galaxies because of their smaller area. The Saracco et al. (1999) surface density is probably lower in this range because of small number statistics as they also use the Persson et al. (1998) standards and aperture magnitudes, and the agreement appears to improve at fainter magnitudes where they have a larger sample. All the $J$ counts in the range 16-20.5 mag have essentially the same slope as these data, $\alpha \sim 0.54$.

The slope of the $H$ band counts in Figure 5 over the $16.5-19$ mag range is $\alpha=0.47$. These counts overlap the brightest magnitude bin from Yan et al. (1998), but the surface density versus magnitude relation shows an apparent "break" at $H=19-20$ mag. The Yan et al. (1998) measurements are based on NICMOS parallel data with Camera 3 over a total area of $8.7 \mathrm{arcmin}^{2}$, and therefore they have relatively few galaxies in the region of overlap. However, the additional NICMOS parallel data studied by Teplitz et al. (1998), which include images from Camera 2 and extend several magnitudes brighter, also show an apparent break at $H=19-20$ mag. Teplitz et al. (1998) transformed several deep $K$ galaxy counts studies to the NICMOS F160W filter, and these transformed $K$ counts appear to match the break in the $H$ galaxy numbermagnitude relation. This break may therefore represent the change in slope seen in $K$ band counts at $K \sim 20 \mathrm{mag}$.

Most published NIR number counts to date have been obtained in the $K$ band (Gardner et al. 1993; Cowie et al. 1994; Glazebrook et al. 1994; Soifer et al. 1994; Djorgovski et al. 1995; McLeod et al. 1995; Moustakas et al. 1997; Minezaki et al. 1998b; Szokoly et al. 1998; Bershady et al. 1998; Saracco et al. 1999; Väisänen et al. 2000; McCracken et al. 2000). Gardner et al. (1996) and Väisänen et al. (2000) have the largest magnitude range of overlap with the DMS counts shown in Figure 6, and the number-magnitude relations from these studies are in good agreement. The slope of the $K$ counts from $14-18 \mathrm{mag}$ is $\alpha=0.54$. At fainter magnitudes there is a great deal of dispersion in the surface 

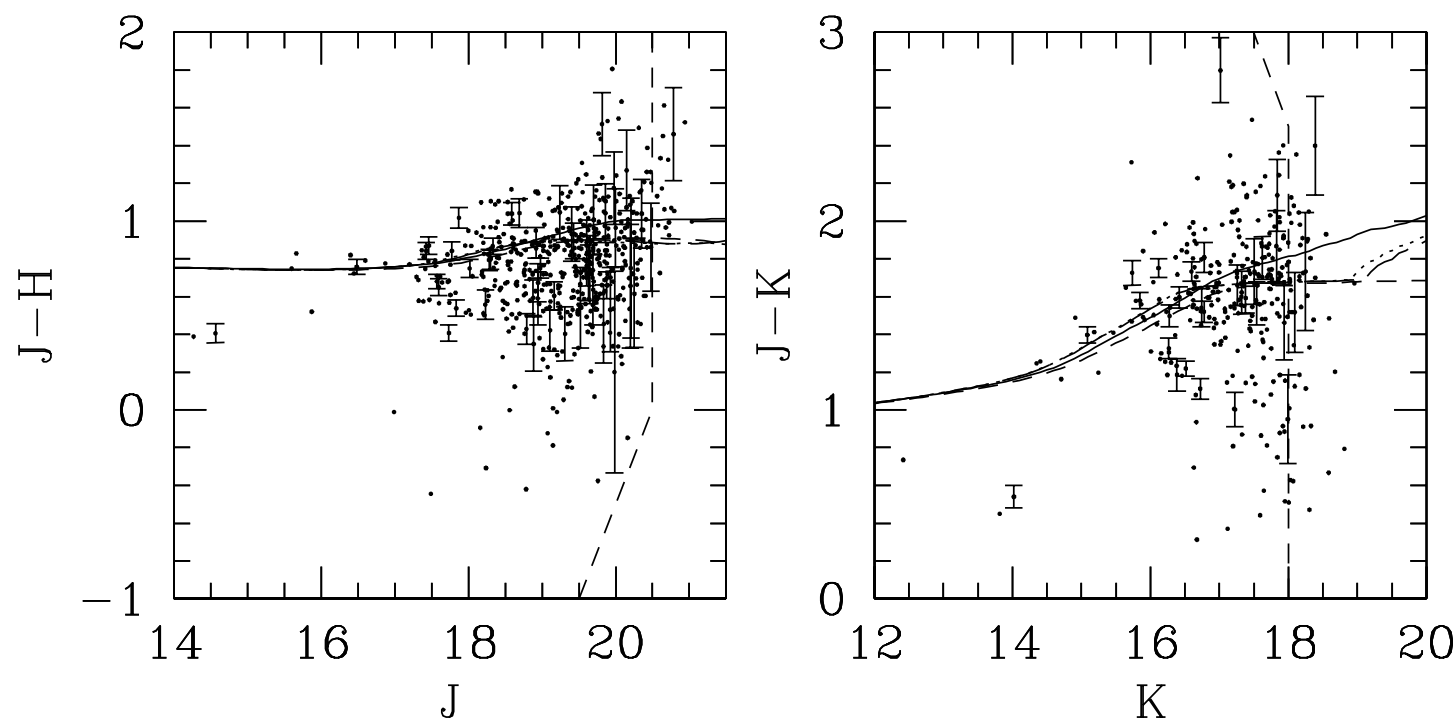

Fig. 7.- Mean near-infrared colors vs. magnitude for the galaxies in this sample. The data are represented by points with or without error bars. For clarity, only every tenth point has an associated error bar. The curves represent the mean colors vs. magnitude for the four models shown in Figs. 4-6 and discussed in $\S 7$. The dashed lines represent the average $50 \%$ limiting magnitudes and colors for an exponential disk with $r_{h}=0$ ". 75 . The scatter of the data points about the model lines is primarily due to variance in the typical galaxy colors rather than photometric uncertainty.

density measurements, though given the small areas of these surveys they are generally consistent with one another. As noted by several authors (e.g., Bershady et al. 1998; Saracco et al. 1999), this dispersion may also be due to different measurement techniques, variations in magnitude systems, and filters, in addition to small number statistics and cosmic variance. The most significant differences are likely due to the method of magnitude measurement, completeness corrections, and, at least at bright magnitudes, star-galaxy separation. For the faint counts presented in Figure 6, Saracco et al. (1999) and Moustakas et al. (1997) apply a uniform galaxy aperture correction for all objects, Bershady et al. (1998) compute completeness and a galaxy aperture correction as a function of object size, Minezaki et al. (1998b) use FOCAS TOTAL magnitudes, and Djorgovski et al. (1995) use only a stellar aperture correction.

I computed the four models shown in Figures 4-6 with NCMOD, a publicly available code for computing galaxy number counts and colors by Gardner (1998). The models correspond to no evolution (solid lines), passive evolution (dotted lines), merging plus passive evolution (short-dashed lines), and dust plus passive evolution (long-dashed lines). For all the models I have assumed $\Omega_{M}=0.3$ and $\Omega_{\Lambda}=0.7$, in agreement with results for Type Ia supernovae (Riess et al. 1998; Perlmutter et al. 1999), the Lyman $\alpha$ forest, and COBE-DMR (Phillips et al. 2001), as well as with number counts in the Hubble Deep Field (Totani \& Yoshii 2000). I chose to fix the cosmological parameters as the uncertainties in $\Omega_{M}$ and $\Omega_{\Lambda}$ are less than the uncertainties in models of galaxy evolution.

All these models are based on the $K$ luminosity function and mix of galaxy types from Gardner et al. (1997) and on the galaxy spectral energy distributions from GISSEL96 (Bruzual \& Charlot 1993). To compute these models I also used the filter transmission curves for the TIFKAM filters plus a measurement of the atmospheric transmission. The "no evolution" model is a pure $k$-correction model. In the passive evolution model, all galaxies form at $z=15$ except for those of the latest galaxy type, which have constant star formation and are always 1 Gyr old. The merging model has the same galaxy evolution as the passive evolution model, but it also includes number evolution in the form $\phi^{*} \propto(1+z)^{\eta}$, with $\eta=1.5$ and conservation of luminosity density. This model is based on the parameterization of Rocca-Volmerange \& Guiderdoni (1990), and it produces similar results to the Broadhurst, Ellis, \& Glazebrook (1992) merging model. This value of $\eta$ is higher than the constraint $\eta \leq 1$ derived by Totani \& Yoshii (2000) in their $\Lambda$ model for the Hubble Deep Field, but it illustrates the effect of adding merging to passive evolution. Finally, the dust model also contains the same galaxy evolution parameterization as the passive evolution model, but with the addition of dust in the plane of the galaxies. This model, based on the work of Bruzual, Margis, \& Calvert (1988) and Wang (1991), was introduced to number count models to correct for their tendency to overproduce the UV flux in galaxies, though it has a minimal effect on the NIR numbermagnitude relation.

The $J, H$, and $K$ observations presented here all agree well with the passive evolution model. They are less consistent with the number density evolution we assumed in the merging model and clearly have a higher surface density of objects than the "no evolution prediction." The observed $J$ counts are all higher than the models, but this may be due to the extrapolation of the $K$ galaxy luminosity function to the $J$ band. As noted above, the HST counts for $H>20$ mag (Teplitz et al. 1998; Yan et al. 1998) have systematically lower surface density. This difference does not appear to be due to differences in the transmission profiles of the two filters. When I calculated the model galaxy counts with the F160W transmission profile, rather than with the $H$ filter, the F160W galaxy count models were offset by less than 0.1 mag fainter than the ground-based $H$ model counts, whereas an offset of $0.3-0.4 \mathrm{mag}$ is needed to explain the difference in surface density.

\section{MEAN COLORS}

Color as a function of apparent magnitude can provide an additional constraint on galaxy evolution models, particularly when several colors are available. I have used the 
Gardner (1998) model to compute the median $J-H$ color versus $J$ magnitude and the median $J-K$ color versus $K$ magnitude for the four models discussed above. Figure 7 shows these two color-magnitude relations for all the galaxies detected in these filters. For the magnitude range of this survey, all these models predict similar median colors and they agree well with the data and $J-K$ colors reported by Bershady et al. (1998) and Saracco et al. (1999) in the region of overlap.

To these apparent magnitude limits, NIR colors do not provide a strong constraint on galaxy evolution as most galaxies are low-redshift $(z<1)$ objects. In this redshift range, the $J, H$, and $K$ filters all sample the old stellar population, which has a relatively flat spectral energy distribution independent of galaxy type. The overlap of the four models and the lack of change in the median color with apparent magnitude reflects the similarity of these galaxies at NIR wavelengths. Bershady et al. (1998) and Saracco et al. (1999) also report $J-K$ colors versus $K$, and my results agree well with their colors.

\section{SUMMARY}

I have presented NIR $J, H$, and $K$ galaxy counts and colors and described in detail the detection efficiencies and selection effects that affect this survey. I have studied several popular methods for measuring the integrated brightness of galaxies as a function of apparent magnitude and found that only methods using aperture magnitudes miss a consistent fraction of a galaxy's light as a function of apparent magnitude for a range of galaxy sizes and profile shapes. This survey is the largest area observed to these depths in all three NIR filters that has been published to date. The $J$, $H$, and $K$ number-magnitude relations and colors are in good agreement with a simple model of passive galaxy evol- ution with at most a small amount $(\eta \leq 1.5)$ of merging in a cosmology with $\Omega_{M}=0.3$ and $\Omega_{\Lambda}=0.7$.

Current model predictions for the number-magnitude relation at NIR wavelengths are very similar to one another, and larger surveys at the faintest magnitudes are needed to discriminate between different models with this diagnostic tool. The models shown in Figures 4-6 suggest that number counts to $H \sim 24 \mathrm{mag}$ or $K \sim 23 \mathrm{mag}$ are necessary to distinguish between the passive evolution and merging model. High angular resolution imaging to measure the mix of galaxy sizes and morphological types as a function of brightness, as well as color information, could prove to be a more efficient means to discriminate between galaxy formation and evolution models with deep, NIR imaging surveys.

I would like to thank Darren DePoy, Patrick Osmer, and David Weinberg for helpful discussions and suggestions, as well as the referee, Jonathan Gardner, for helpful comments that have improved this presentation. I am grateful to Christopher J. Burke and Adam Steed for assistance with some of the observations and to Mike Lannon for supplying the TIFKAM filter curves in digital form. I would also like to thank the MDM staff for their support and the Ohio State University TAC for a generous allocation of telescope time. I was supported in part by a Presidential Fellowship from Ohio State University and received additional travel and other support from a PEGS grant and the Department of Astronomy at Ohio State University. TIFKAM was funded by the Ohio State University, the MDM consortium, MIT, and NSF grant AST 96-05012. NOAO and USNO paid for the development of the ALADDIN arrays and contributed the array currently in use in TIFKAM.
Bershady, M. A., Lowenthal, J. D., \& Koo, D. C. 1998, ApJ, 505, 50 Bertin, E., \& Arnouts, S. 1996, A\&AS, 117, 393

Brinchmann, J., \& Ellis, R. S. 2000, ApJ, 536, L77

Broadhurst, T. J., Ellis, R. S., \& Glazebrook, K. 1992, Nature, 355, 55

Bruzual, A. G., \& Charlot, S. 1993, ApJ, 405, 538

Bruzual, A. G., Margis, C. G., \& Calvert, N. 1988, ApJ, 333, 673

Cowie, L. L., Gardner, J. P., Hu, E. M., Songaila, A., Hodapp, K.-W., \& Wainscoat, R. J. 1994, ApJ, 434, 114

Cowie, L. L., Songaila, A., Hu, E. M., \& Cohen, J. G. 1996, AJ, 112, 839

Dalcanton, J. J. 1998a, ApJ, 495, 251

.1998b, AJ, submitted

Davies, J. I. 1990, MNRAS, 244, 8

Disney, M. 1976, Nature, 263, 573

Disney, M., \& Phillipps, S. 1983, MNRAS, 205, 1253

Djorgovski, S., et al. 1995, ApJ, 438, L13

Elias, J. H., Frogel, J. A., Matthews, K., \& Neugebauer, G. 1982, AJ, 87, 1029

Ellis, R. S. 1997, ARA\&A, 35, 389

Frogel, J. A. 1998, PASP, 110, 200

Gardner, J. P. 1995, ApJS, 98, 441

. 1998, PASP, 110, 291

Gardner, J. P., Cowie, L. L., \& Wainscoat, R. J. 1993, ApJ, 415, L9

Gardner, J. P., Sharples, R. M., Carrasco, B. E., \& Frenck, C. S. 1996, MNRAS, 282, L1

Gardner, J. P., Sharples, R. M., Frenk, C. S., \& Carrasco, B. E. 1997, ApJ, 480, L99

Gehrels, N. 1986, ApJ, 303, 336

Glazebrook, K., Peacock, J. A., Collins, C. A., \& Miller, L. 1994, MNRAS, 266,65

Gould, A. 1995, ApJ, 440, 510

Guiderdoni, B., \& Rocca-Volmerange, B. 1990, A\&A, 227, 362

Hall, P. B., Green, R. F., \& Cohen, M. 1998, ApJS, 119, 1

Hall, P. B., Osmer, P. S., Green, R. F., Porter, A. C., \& Warren, S. J. 1996a, ApJS, 104, 185

.1996b, ApJ, 471, 1073

Hubble, E. 1934, ApJ, 79, 8

Im, M., Casertano, S., Griffiths, R. E., Ratnatunga, K. U., \& Tyson, J. A. 1995, ApJ, 441, 494

\section{REFERENCES}

Jarvis, J. F., \& Tyson, J. A. 1981, AJ, 86, 476

Kennefick, J. D., Osmer, P. S., Hall, P. B., \& Green, R. F. 1997, AJ, 114, 2269

Koo, D. C., \& Kron, R. G. 1992, ARA\&A, 30, 613

Kron, R. G. 1980, ApJS, 43, 305

Lilly, S., et al. 1998, ApJ, 500, 75

Liu, C. T., Green, R. F., Hall, P. B., \& Osmer, P. S. 1998, AJ, 116, 1082

Martini, P. 2001, AJ, submitted (astro-ph/0009287)

Martini, P., \& Osmer, P. S. 1998, AJ, 116, 2513

McCracken, H. J., Metcalf, N., Shanks, T., Campos, A., Gardner, J. P., \& Fong, R. 2000, MNRAS, 311, 707

McLeod, B. A., Bernstein, G. M., Rieke, M. J., Tollestrup, E. V., \& Fazio, G. G. 1995, ApJS, 96, 117

Minezaki, T., Cohen, M., Kobayashi, Y., Yoshii, Y., \& Peterson, B. A. 1998a, AJ, 115, 229

Minezaki, T., Kobayashi, Y., Yoshii, Y., \& Peterson, B. A. 1998b, ApJ, 494, 111

Moffat, A. F. J. 1969, A\&A, 3, 455

Moustakas, L. A., Davis, M., Graham, J. R., Silk, J., Beterson, B. A., \& Yoshii, Y. 1997, ApJ, 475, 445

Osmer, P. S., Kennefick, J. D., Hall, P. B., \& Green, R. F. 1998, ApJS, 119, 189

Perlmutter, S., et al. 1999, ApJ, 517, 565

Persson, S. E., Murphy, D. C., Krzeminski, W., Roth, M., \& Rieke, M. J. 1998, AJ, 116, 2475

Petrosian, V. 1976, ApJ, 209, L1

-. 1998, ApJ, 507, 1

Phillipps, S., Davies, J. I., \& Disney, M. J. 1990, MNRAS, 242, 235

Phillips, J., Croft, R. A. C., Weinberg, D. H., Hernquist, L., Katz, N., \& Pettini, M. 2001, ApJ, submitted (astro-ph/0001089)

Pogge, R. W., et al. 1998, Proc. SPIE, 3354, 414

Riess, A. G., et al. 1998, AJ, 116, 1009

Rocca-Volmerange, B., \& Guiderdoni, B. 1990, MNRAS, 247, 166

Roche, N., Ratnatunga, K., Griffiths, R. E., \& Im, M. 1997, MNRAS, 288, 200

Roche, N., Ratnatunga, K., Griffiths, R. E., Im, M., \& Naim, A. 1998, MNRAS, 293, 157

Sandage, A. 1961, ApJ, 133, 355 
Sandage, A., \& Perelmuter, J.-M. 1990, ApJ, 350, 481

Saracco, P., D'Odorico, S., Moorwood, A., Buzzoni, A., Cuby, J.-G., \& Lidman, C. 1999, A\&A, 349, 751

Smail, I., Hogg, D. W., Yan, L., \& Cohen, J. G. 1995, ApJ, 449, L105

Soifer, B. T., et al. 1994, ApJ, 420, L1

Szokoly, G. P., Subbarao, M. U., Connolly, A. J., \& Mobasher, B. 1998, ApJ, 492, 452

Teplitz, H. I., Gardner, J. P., Malumuth, E. M., \& Heap, S. R. 1998, ApJ, 507, L17

Teplitz, H. I., McLean, I. S., \& Malkan, M. A. 1999, ApJ, 520, 469
Thompson, D., et al. 1999, ApJ, 523, 100

Tinsley, B. M. 1977, ApJ, 211, 621

Totani, T., \& Yoshii, Y. 2000, ApJ, 540, 8

Väisänen, P., Tollestrup, E. V., Willner, S. P., \& Cohen, M. 2000, ApJ, 540, 593 (erratum 543, 502)

Valdes, F. 1982, Proc. SPIE, 331, 465

Wang, B. 1991, ApJ, 383, L37

Yan, L., McCarthy, P. J., Storrie-Lombardi, L. J., \& Weymann, R. J. 1998, ApJ, 503, L19

Yoshii, Y., \& Takahara, F. 1988, ApJ, 326, 1 